\title{
Una aproximación al manejo de cuencas hidrográficas desde el ordenamiento territorial en la Región Metropolitana de Buenos Aires
}

\author{
An approach to drainage basin management from spatial planning in the \\ Metropolitan Region of Buenos Aires
}

\author{
Santiago Báez \\ baez.santi93@gmail.com \\ Centro de Investigaciones Geográficas / Instituto de \\ Investigaciones en Humanidades y Ciencias Sociales \\ (CONICET/ UNLP) / Facultad de Humanidades \\ y Ciencias de la Educación. Universidad Nacional \\ de La Plata, Argentina.

\section{Ludmila Cortizas} \\ ludmi.cortizas@gmail.com \\ Laboratorio de Investigaciones del Territorio y el \\ Ambiente (LINTA, CIC-PBA). / Departamento de \\ Geografía (FaHCE-UNLP) / Centro \\ de Investigaciones Geográficas, Instituto de \\ Investigaciones en Humanidades y Ciencias \\ Sociales ( CIG, IdIHCS), Argentina
}

Recepción: 02 Julio 2021

Aprobación: 31 Agosto 2021

Publicación: 01 Noviembre 2021

Cita sugerida: Báez, S. y Cortizas, L. (2021). Una aproximación al manejo de cuencas hidrográficas desde el ordenamiento territorial en la Región Metropolitana de Buenos Aire. Geograficando, 17(2), e104.

https://doi.org/10.24215/2346898Xe104
Resumen: En este trabajo se propone discutir sobre aquellas conceptualizaciones vinculadas al manejo o gestión de cuenca y al ordenamiento territorial, para luego indagar cómo estas miradas permean los instrumentos y organismos de ordenamiento territorial -actualmente vigentes- que tienen influencia en la Región Metropolitana de Buenos Aires (RMBA). Desde el punto de vista metodológico, se recurrió a la recopilación y análisis de aquellos instrumentos y organismos más representativos que tienen influencia en la RMBA, bibliografía específica, informes técnicos y sitios web oficiales. Los resultados obtenidos evidencian que aún continúan predominando las concepciones más tradicionales de ordenamiento territorial, manejo y gestión de cuencas, y, a su vez, la complejidad ambiental y territorial de la RMBA pareciera que no termina de ser correctamente abordada por tales instrumentos, dadas las múltiples jurisdicciones y el elevado grado de dispersión normativa existente.

Palabras clave: Ordenamiento territorial, Manejo y Gestión de Cuencas, Instrumentos, Organismos, RMBA.

Abstract: The aim of this paper is to discuss conceptualizations
regarding drainage basin management and spatial planning, later
to inquire how these viewpoints influence the instruments and
institutions that currently guide the development of such areas
in the Metropolitan Region of Buenos Aires (RMBA, Región
Metropolitana de Buenos Aires). In terms of methodology,
we collated and analyzed the most representative instruments
and institutions which influence the RMBA, including specific
bibliography, technical reports and official websites. The results
show that the traditional conceptualization of both spatial
planning and drainage basin management still predominate. In
addition, the diverse instruments consulted do not seem to
properly attend to the environmental and spatial complexity of
the RMBA, given the latter's multiple jurisdictions and the high
degree of normative dispersion.

Keywords: Spatial Planning, Drainage Basin Management, Instruments, Institutions, RMBA. 


\section{INTRODUCCIÓN}

Desde hace algunas décadas conceptos como el de cuenca, gestión de la misma y ordenamiento territorial han estado en el foco de la discusión de múltiples análisis territoriales, tanto urbanos como rurales. A raíz de la lectura de distintos autores que han trabajado con ellos, en este trabajo se considera que las miradas predominantes sobre ordenamiento territorial y gestión de cuencas corresponden a tipos ideales, modelos que suponen -desde la perspectiva de este trabajo- resultados de muy difícil alcance, y que se pretenden universales, pero que lejos están de aprehender las diversas realidades locales. En este sentido, este trabajo propone discutir sobre aquellas conceptualizaciones vinculadas al manejo o gestión de cuenca y al ordenamiento territorial, para luego indagar cómo estas miradas permean los instrumentos y organismos actualmente vigentes de ordenamiento territorial que tienen influencia en la Región Metropolitana de Buenos Aires -en adelante RMBA-. Se trata de un conglomerado urbano integrado por la Ciudad Autónoma de Buenos Aires -en adelante CABA- y 40 partidos (DPOUyT, 2007), el cual cuenta, según el Censo de 2010, con 14.819.137 habitantes y abarca $13.947 \mathrm{~km} 2$ (Lanfanchi, et al., 2017).

La elección del área de estudio tiene que ver con una complejidad que le es propia, tanto desde el punto de vista social como natural. El aglomerado urbano más grande del país posee la particularidad de relacionarse con grandes cuencas hidrográficas; allí, la multiplicidad de procesos, actores e intereses vinculados a las diversas jurisdicciones configuran un escenario complejo. Es por ello que las preguntas que guían la investigación son: ¿cómo se concibe el ordenamiento territorial en los instrumentos de gestión que involucran a la RMBA?, ¿cómo son contempladas las cuencas hidrográficas en los mismos?, ¿qué visión respecto al manejo o gestión de cuencas manifiestan aquellos organismos de la RMBA creados para tal fin?, ¿qué procesos se toman en consideración y qué metas se proponen alcanzar? Para responder a estas preguntas se seleccionaron aquellos instrumentos y organismos actualmente vigentes, más representativos y con influencia directa en la RMBA. A su vez, se recurrió a la búsqueda, recopilación y análisis de bibliografía específica, de normativa nacional y provincial vinculada a la temática, la lectura de informes técnicos y la revisión de sitios web oficiales.

\section{Ordenamiento territorial Y Manejo De Cuencas. Entre sus acepciones Y APLICACIONES}

Existen, en la literatura académica, múltiples interpretaciones y formas de definir diversos conceptos. Esto se debe, generalmente, a que coexisten diferentes escuelas de pensamiento que los abordan con distintas temporalidades y espacialidades, y reciben aportes de múltiples ciencias. En primer lugar, se recuperan aquellas conceptualizaciones y perspectivas sobre el ordenamiento territorial, que se trabajan desde hace años y en distintos países. Algunos autores (Zoido, 1998; Montes Lira, 2001) se refieren al ordenamiento del territorio como un instrumento basado en la voluntad y acción pública materializada en una política compleja, fundada en instrumentos jurídicos y prácticas administrativas. Además, implica una visión prospectiva, un modelo territorial deseable (Massiris Cabeza, 2005), de contenidos específicos y de acciones concretas desarrolladas, es decir, como un proceso vinculado a un programa de acción territorial. Montes Lira (2001) lo enmarca en la planificación estratégica, e incorpora la eficiencia, la participación y la flexibilidad.

Por su parte, Gómez Orea (2003), Massiris Cabeza (2005), y Farinos Dasí (2014) incorporan las nociones de organización, disciplina técnica, regulación y corrección de los problemas y desequilibrios territoriales en las diferentes escalas. Según estos autores, el territorio ha de requerir un "orden", acorde a sus potencialidades y limitaciones, con las expectativas y las aspiraciones de la población y con los objetivos de desarrollo presentes. Cabrales Barajas (2006) reconoce las múltiples dimensiones del término, y, a su vez, coincide en que está íntimamente relacionado a los vaivenes de los modelos de desarrollo vigentes. 
Los planteos conceptuales presentados en los párrafos precedentes vendrían a formar parte de lo que Arzeno (2019) denomina como "miradas predominantes del ordenamiento territorial". Al retomar sus definiciones y los términos utilizados, la autora se pregunta si verdaderamente es posible hablar de armonía, aspiraciones comunes de la población, orden y organización, si se consideran las diferencias existentes en el territorio y los conflictos que lo caracterizan. En este sentido, Arzeno (2019) establece que estas perspectivas del ordenamiento territorial ven los conflictos por el territorio como expresión de un desorden que requiere de la intervención estatal, y como instancias negociables que deben ser consensuadas a partir de la creación de espacios participativos y gestionados de manera eficiente por este actor social. El Estado, en la mayoría de los escritos, es visto como una entidad eficaz y capaz de garantizar un ordenamiento funcional a los intereses generales de la sociedad. No obstante, también se reconoce que en el territorio y en el espacio urbano en particular, el orden beneficia a unos y perjudica a otros. Es por ello que las propuestas y políticas estatales de ordenamiento del territorio requieren de un análisis crítico que coloque el énfasis en las conflictividades, las relaciones de poder y las lógicas de los actores, de cara a pensar otros ordenamientos posibles.

Dentro del universo del ordenamiento territorial y las distintas escalas de instrumentación existen experiencias que utilizan como unidad territorial la cuenca hidrográfica como parte de procesos de gestión o manejo de estas. A decir de González y Natenzon (2002), la cuenca hidrográfica es un recorte territorial específico en el que se entretejen procesos de índole natural y procesos sociales. Esta definición complejiza la conceptualización más tradicional y física de las cuencas hidrográficas, que las define como áreas cóncavas en las que se desarrolla un sistema fluvial, y que involucran una determinada superficie terrestre y procesos hídricos de dirección establecida por las diferencias de altura. De esta forma, se entiende que en una cuenca convergen múltiples intereses con sus propias lógicas contradictorias, que configuran un espacio de alta incertidumbre política y técnica (González y Natenzon, 2002).

De acuerdo a Dourojeanni y Jouravlev (1999), la cuenca hidrográfica es reconocida como la unidad territorial más adecuada para la gestión integrada de los recursos hídricos, universo que a su vez contempla el manejo o gestión de las mismas como una instancia de gestión ambiental. Según los autores, el manejo de cuencas hidrográficas nace de la traducción literal del término management, que en español tiene hasta cuatro acepciones: gestión, administración, ordenamiento y manejo. El enfoque clásico de manejo de cuencas proviene de Estados Unidos y es concebido en el sentido de "manejarla" o "manipularla" para regular la descarga de agua. No obstante, los autores establecen que ello se inscribe como una actividad mixta que combina aspectos de protección, conservación y aprovechamiento de recursos. Desde una visión similar, Alvino (2012) plantea la multidimensionalidad del concepto y piensa al manejo de cuencas como un proceso, como un sistema y como un conjunto de actividades organizadas secuencialmente, que toma a la cuenca como unidad para el análisis, la planificación y la gestión ambiental. Sin embargo, al igual que la noción de territorio, ese proceso no está exento de confrontaciones, en tanto las relaciones entre diversos actores suponen la existencia de conflictos. Dourojeanni y Jouravlev (1999) plantean que para llevar adelante procesos de gestión o manejo integrado de cuencas se deben realizar alianzas o concertaciones entre múltiples actores que normalmente actúan en forma sectorializada. Estas relaciones conflictivas muchas veces se traducen en medidas que pueden beneficiar y también producir consecuencias negativas a algunos actores, y, a su vez, pueden incurrir en un factor desencadenante o potenciador de problemas ambientales.

En síntesis, los conceptos de ordenamiento territorial y manejo de cuencas denotan múltiples formas de comprensión y definición que corresponden a miradas predominantes y frecuentes en la literatura académica. Sin embargo, al considerar la complejidad del territorio y su conflictividad inherente, se reconoce que dichas definiciones están lejos de ser capaces de aprehender las diversas realidades locales y, en particular, la de la región estudiada en este trabajo, lo cual requiere de una perspectiva crítica para su abordaje, que contemple la multidimensionalidad y la existencia de actores que despliegan sus lógicas en a la construcción del territorio. 


\section{Ordenamiento territorial y manejo de cuencas en América Latina y Argentina}

Al realizar un recorrido por algunos trabajos de América Latina (Ugalde, 2010; Hernández Peña, 2010; Carvajal Sánchez, 2011; Ruiz Rivera, et al., 2015; Haesbaert, 2014; entre otros) se observa que diversas nociones de estas conceptualizaciones han sido incorporadas en la política de ordenamiento territorial. Dentro de las experiencias vinculadas a la temática, también se ha considerado la cuenca hidrográfica como unidad de planificación, lo que también ha suscitado diversas investigaciones en varios países (véase Pulido, 2011; Nené-Preciado, et al., 2017; Braz, et al., 2020; entre otros). Cabrales Barajas (2006), por caso, plantea que estos enfoques que contemplan la cuenca como unidad física de organización de actividades económicas y asentamientos humanos tienen como principal referente a la experiencia de la Autoridad del Valle del Tennessee (TVA por sus siglas en inglés). Dicho modelo ha sido replicado en América Latina (en países como Perú, Brasil y México) a partir de la década de 1940, mediante la creación de comisiones y corporaciones que representan esfuerzos por gestionar cuencas hidrográficas en línea con objetivos de desarrollo. Sin embargo, pocos casos resultaron exitosos, y el formato de intervención territorial quedó restringido en su mayoría a una acción sectorial de gestión de recursos naturales. Esto se debió a que en la práctica la política de cuencas hidrográficas fue considerada como un simple sustrato físico y proveedor de recursos naturales, sin lograr el ensamblaje con aspectos sociales (Cabrales Barajas, 2006).

En Argentina, Reese (2006) plantea que existe una rigidez común en los organismos estatales responsables de la planificación y de los proyectos urbanos en términos de crear e implementar nuevos instrumentos normativos, ya que la política urbanística es concebida únicamente como una acción regulatoria a través de códigos u ordenanzas de zonificación y/o edificación. Asimismo, el autor también nota una desarticulación entre la formulación de planes y proyectos, la habilitación de herramientas fiscales y administrativas, y el dictado de políticas económicas activas que favorezcan/desalienten ciertas actividades o procesos específicos en el ordenamiento del territorio. Entre algunos esfuerzos vinculados a la temática se destaca la firma de gobernadores y autoridades nacionales del Acta Base para la creación del Consejo Federal de Planificación y Ordenamiento Territorial (COFEPLAN) en 2008, organismo que presentó un anteproyecto de ley de ordenamiento territorial, el cual no prosperó en reiteradas ocasiones. Este organismo fue creado con el objetivo de participar en la planificación, articulación y armonización de las políticas de planificación y ordenamiento territorial, dirigidas a lograr un país equilibrado, integrado, sustentable y socialmente justo.

El Plan Estratégico Territorial (PET) es quizás el instrumento por excelencia que guía la planificación territorial en el país, entendida como una herramienta que pretende dotar de racionalidad a los procesos de decisión política de las acciones del gobierno en sus tres niveles. Sus lineamientos le dan un marco a las experiencias de ordenamiento territorial en las escalas inferiores, ya que guía el despliegue de la inversión pública y privada, con el objeto de construir un país integrado, equilibrado, sustentable y socialmente inclusivo, que permita alcanzar un elevado nivel de desarrollo humano, incrementar la competitividad del país y mejorar la calidad de vida de sus habitantes (PET, 2018). El instrumento incorpora la Nueva Agenda Urbana creada en la reunión de Hábitat III en Quito (2016), la cual posee una visión multiescalar de la planificación a través de la cual busca integrar las acciones de los gobiernos municipales, fomentar las capacitaciones y favorecer a la participación de los distintos actores locales en la planificación y gestión del territorio (PET, 2018).

En lo que respecta al manejo de cuencas, Casaza (2004) reconoce que aún no existen criterios comunes para definir qué se entiende por dicho concepto en Argentina, y tampoco se han aunado criterios para la adopción de una determinada terminología. Asimismo, la autora plantea que el concepto evolucionó con los años; parte desde de la noción de ordenación de las vertientes, para luego dar lugar a una visión predominante sobre la administración de cuencas, vinculada al manejo del agua y su aprovechamiento a partir de la construcción de obras hidráulicas para el desarrollo y la ocupación de territorios. También, desde este abordaje, se buscó resolver problemas hidrológicos, de riesgo hídrico y de conservación de suelos. Según la autora, los años 
posteriores a los 90 estuvieron muy influidos por el desarrollo sustentable, la visión sistémica y los nuevos paradigmas de participación social, por lo que al hablar de cuenca no solo se hacía referencia al sistema natural, sino que se pensaba en interacción con el sistema socioeconómico (Casaza, 2004).

Es importante destacar que uno de los actores más influyentes en el manejo y gestión de cuencas, y a su vez en el ordenamiento del territorio, es el Estado, a través de legislaciones y de la implementación de políticas públicas. Estas son entendidas como la toma de posición del Estado, que implica la adopción de un conjunto de acciones y omisiones, frente a una cuestión socialmente problematizada en un determinado momento y lugar (Oszlak y O’Donnell, 1995). Esta intervención posee, según los autores antes citados, una cierta direccionalidad y una determinada orientación normativa que afecta el desarrollo y desenvolvimiento del problema en cuestión. En relación a esto, Haesbaert (2014) plantea que todo territorio implica algún tipo de control y de imposición de un determinado grado o nivel de orden, que va a estar dado por la prevalencia de prácticas políticas de transformación del espacio impulsadas por distintos actores, tanto hegemónicos como contrahegemónicos. Según este autor, son las distintas dinámicas económicas, políticas, culturales y naturales las que se conjugan en contextos determinados y dan lugar a resultantes que dificultan la construcción de políticas de ordenamiento territorial (Haesbaert, 2006).

En un trabajo reciente, Calderón et al. (2020) analizaron la vinculación de los aspectos normativos e institucionales entre las políticas públicas de ordenamiento territorial y la gestión del agua en Argentina y en la provincia de Buenos Aires. De dicho análisis, los autores destacan que a nivel nacional hay una ausencia de leyes específicas vinculadas al ordenamiento territorial y a la gestión de aguas (temáticas consideradas tanto separadas como una temática única), aunque reconocen un avance en materia de política hídrica con la formulación de los Principios Rectores de la Política Hídrica (2003). Más aún, plantean que no se ha avanzado con la creación de instrumentos que vinculen el ordenamiento territorial y la gestión del agua a nivel institucional y/o normativo, siendo la gestión del riesgo la única temática abordada hasta la fecha, cuya máxima expresión fue la sanción de la ley nacional que creó el Sistema Nacional de Gestión del Riesgo (Zapperi, 2018; Calderón et al., 2020). Cabe destacar también la sanción de la Ley General del Ambiente (Ley Nacional $\left.N^{\circ} 25.675\right)$ en el año 2002, la cual establece los presupuestos mínimos para la gestión, preservación y protección del ambiente. También en el año 2002 el Congreso Nacional aprobó el Régimen de Gestión Ambiental de Aguas (Ley Nacional $N^{\circ} 25.688$ ), pero su reglamentación aún sigue pendiente, lo cual es foco de reclamo por parte de distintas organizaciones ambientales y sociales.

Por su parte, en el plano institucional es importante mencionar que la coordinación e implementación de políticas públicas referidas a los recursos hídricos en el territorio nacional se encuentra a cargo de la Secretaría de Infraestructura y Política Hídrica, la cual también supervisa y coordina el accionar de distintos organismos bajo su órbita. Concibe a la gestión hídrica como el resultado de múltiples decisiones, públicas y privadas, que son tomadas en forma independiente, por lo cual resulta imperioso coordinar entre quienes toman las decisiones para lograr una gestión adecuada. Es por ello que promueve la institucionalización y el fortalecimiento de organizaciones (comités) de cuenca interjurisdiccionales.

Otro organismo que opera en paralelo es el Consejo Hídrico Federal (COHIFE) concebido como instancia federal para el tratamiento de los aspectos de carácter global, estratégico, interjurisdiccional e internacional de los recursos hídricos. En este sentido, la institución reconoce que la figura del comité de cuenca interjurisdiccional es clave, en tanto que representa una instancia de negociación en la cual se pueden llegar a acuerdos entre distintas jurisdicciones respecto a cuestiones vinculadas a la gestión del agua. Este organismo dio origen formal a los Principios Rectores de la Política Hídrica (PRPH), los cuales brindan el marco para promover el desarrollo armónico e integral del país en materia del agua, y tienen como eje la gestión integrada de la misma. Desde estos, se apunta a lograr la gestión conjunta de la cantidad y calidad del agua mediante la actualización y armonización de las normativas y la coordinación intersectorial tendiente a articular la gestión hídrica con la gestión ambiental, dentro de las disposiciones del marco constitucional vigente. Por otro lado, la cuenca hidrográfica es considerada como la unidad territorial más 
apta para la planificación y gestión coordinada de los recursos hídricos ( $\left.\mathrm{PR} \mathrm{N}^{\circ} 19\right)$, y es por ello que ve en los comités de cuenca espacios de discusión, conciliación, concertación, coordinación y cogestión del agua (PR $\left.\mathrm{N}^{\circ} 25\right)$. En esta línea, resulta importante destacar que Argentina cuenta con 16 comités de cuenca interprovinciales, mientras que también existen múltiples organismos de estas características al interior de las distintas provincias.

La aproximación realizada hasta este punto, referida al ordenamiento territorial y al manejo de cuencas hidrográficas en el país, permite observar que son múltiples los instrumentos que influyen en la materia. A su vez, por disposición de la Constitución nacional, las provincias y CABA tienen la potestad de gestionar sus territorios y los recursos naturales presentes en ellos, lo cual incluye la cuestión del manejo de cuencas. A su vez, algunas provincias delegan en los municipios la facultad de llevar adelante la política de ordenamiento territorial de sus partidos/departamentos. La RMBA es un caso particular, dado que sus características trascienden las fronteras políticas de CABA y la provincia de Buenos Aires, y ponen en evidencia la complejidad que esta supone a la hora de pensar cuestiones referidas al ordenamiento territorial y al manejo de cuencas, tema al cual se destina el siguiente apartado.

\section{Instrumentos y ORganismos de Ordenamiento Territorial y Manejo de cuencas EN LA RMBA}

Como puede observarse en la Figura 1, en la porción territorial de la RMBA que corresponde a los partidos de la provincia de Buenos Aires se destacan 14 cuencas hidrográficas, a las cuales se suman las que se encuentran en CABA.

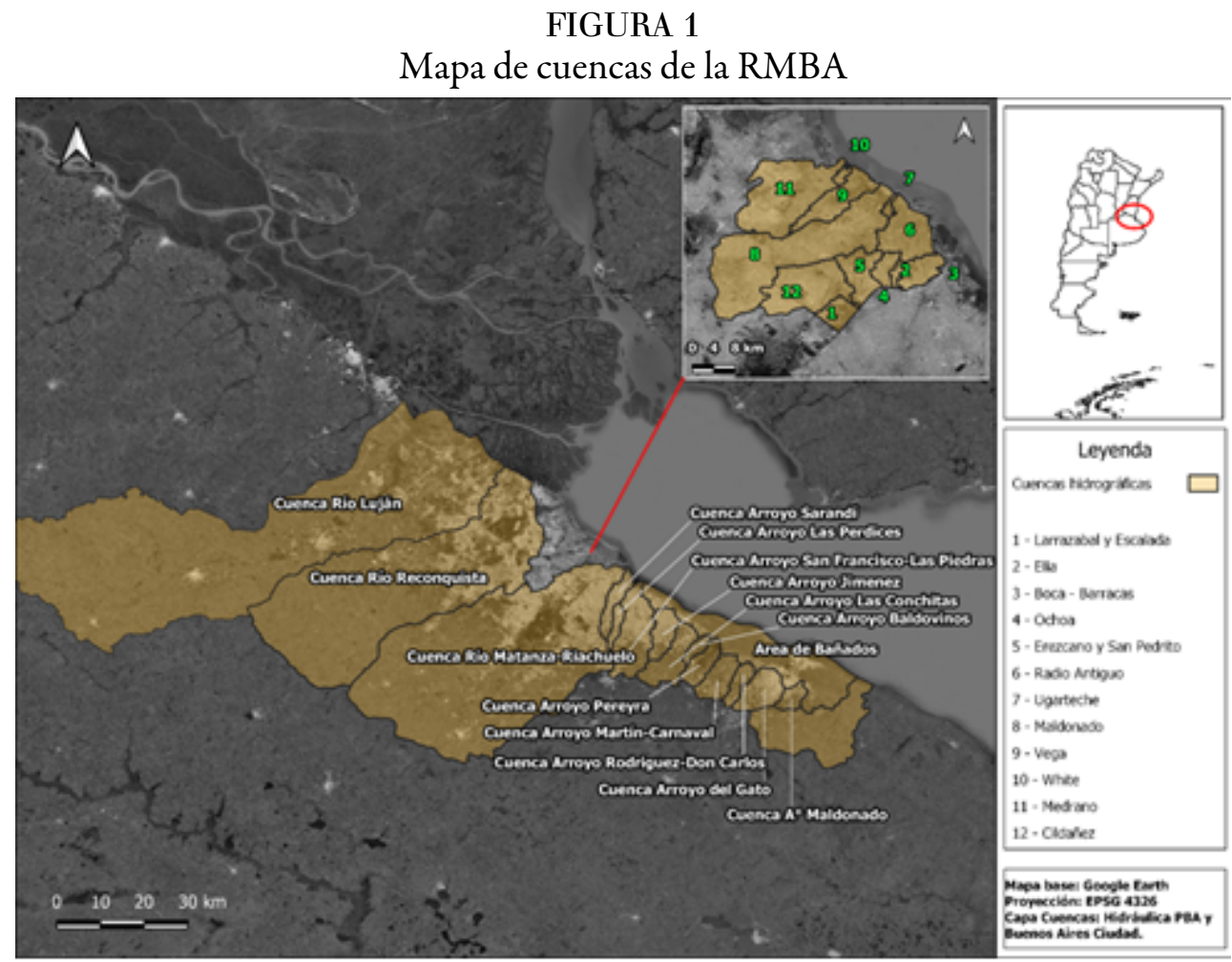

Fuente: Elaboración propia.

De ello se deriva que la RMBA se caracteriza por una integridad funcional y continuidad ambiental que no corresponde a un único espacio de gestión territorial, ya que en esa región coexisten una diversidad de organismos públicos sectoriales, instituciones y diferentes niveles del Estado, que operan con distintos 
alcances e intereses en la construcción del territorio, su ordenamiento y gestión. Más aún, Merlinsky (2006) plantea que las políticas de planeamiento urbano no suelen estar articuladas con las políticas ambientales, lo que da lugar al surgimiento de vacíos legales y a una expansión urbana incontrolada con importantes consecuencias sobre la calidad ambiental. Según Capuccio y Mignaqui (2014), esta cuestión es acompañada también por la superposición y a veces contradicción entre legislaciones y jurisdicciones, a lo que se le suma la complejidad inherente a la toma de decisiones en conjunto y la diversidad de enfoques y signos políticos en las distintas jurisdicciones al momento de llevar adelante la gestión urbana y ambiental del territorio. En este sentido, la falta de coordinación interjurisdiccional, junto con todas estas cuestiones mencionadas, tiene como consecuencia que la gestión ambiental del territorio presente dificultades importantes, situación que se ve replicada en la gestión y manejo integrado de las cuencas hidrográficas (Rotger, 2018).

En esta línea, el PET reconoce a la RMBA como la mayor concentración y convergencia de actividades, población y problemáticas territoriales, e identifica que sus cuencas hidrográficas presentan situaciones diferenciales de degradación ambiental y diversas problemáticas, ligadas a la ocupación y transformación de grandes superficies, entre suelo productivo y áreas ambientalmente críticas (PET, 2018). Por su parte, los Lineamientos Estratégicos para la RMBA (2007) incorporan el enfoque del desarrollo sustentable en el tratamiento de los cursos de agua, según el cual reconocen como parte de la matriz ambiental el manejo de cuencas y el espacio ribereño, el control de los rellenos, la gestión de los residuos, entre otros (Capuccio y Mignaqui, 2014). Así visto, el manejo de cuencas se liga a la prevención y control de efectos ambientales adversos a través de infraestructura y obras hidráulicas, a la organización del uso del suelo y del agua como recurso, a la gestión de redes, calidad de las aguas y control de efluentes, a la restitución de áreas verdes afectadas, y la elaboración de un plan de manejo para cada cuenca junto al ejercicio del poder de policía. El ordenamiento territorial, por su parte, es visto como un instrumento para mejorar la vida cotidiana, que permitiría a la sociedad desarrollarse en un contexto ambiental libre, equilibrado y sustentable.

\subsection{Provincia de Buenos Aires (PBA)}

Además de la injerencia nacional presentada anteriormente, es importante destacar también el rol que posee el gobierno de la provincia de Buenos Aires en la gestión de la RMBA. En términos generales, existen a nivel provincial dos normativas específicas que guían el ordenamiento territorial, por un lado (Decreto ley 8.912/77), y la gestión del agua, por el otro (Ley 12.257/98). El Decreto ley 8.912 de Ordenamiento Territorial y Uso del Suelo del 77 se planteó como un instrumento pensado para atender la necesidad de controlar el rápido y "desordenado" crecimiento que se verificaba en la casi totalidad de las ciudades de la misma (Reese, 2014). Las principales disposiciones que prevé se orientan a lograr el orden urbano a partir de la reglamentación del uso, ocupación y subdivisión del suelo y de niveles de infraestructura y equipamiento para las áreas urbanas (Ríos, 2017), para lo cual delega la responsabilidad del ordenamiento territorial los partidos.

Entre sus objetivos fundamentales se destacan la corrección de los efectos producidos por las acciones degradantes del ambiente, la preservación y el mejoramiento del mismo, una adecuada organización de las actividades en el espacio, y demás. Se pone énfasis en la participación de la comunidad en el proceso de ordenamiento territorial y en la generación de una conciencia comunitaria sobre la necesidad vital de la preservación y recuperación de los valores ambientales. En lo que a las cuencas hidrográficas refiere, se verifica que la normativa no las contempla como unidad territorial.

Por su parte, en la Constitución provincial, se reconoce el dominio ejercido sobre el territorio, el ambiente y sus recursos, para asegurar una gestión ambientalmente adecuada (Art.28). Se enfatiza sobre la preservación, recuperación y conservación de sus recursos, planificar su aprovechamiento racional, controlar el impacto ambiental que las actividades pudiesen provocar, defender el ambiente y garantizar el derecho a recibir 
información adecuada al respecto. Todo ello, para mantener la integridad física, su capacidad productiva, y para resguardar áreas de importancia ambiental.

En esta línea aparece la figura del Organismo Provincial para el Desarrollo Sostenible (OPDS), la autoridad de aplicación de la normativa ambiental de la provincia de Buenos Aires. Este organismo define al ordenamiento ambiental del territorio como un componente fundamental, ineludible e indisociable del ordenamiento territorial. Se trata de un proceso técnico-político-administrativo basado en un uso adecuado del territorio con un enfoque ecosistémico, el cual establece la compatibilidad de las actividades que se realizan en las diferentes unidades territoriales con las características ecológicas, sociales y económicas de las mismas, y que hacen sostenible el desarrollo desde el punto de vista ambiental.

Referido específicamente a los recursos hídricos, en 1998 se sancionó el Código de Aguas de la provincia (Ley 12.257), el cual dispone el régimen de protección, conservación y manejo del recurso hídrico, y estipula los lineamientos para la creación de la Autoridad del Agua (ADA), organismo que funciona como autoridad de aplicación de la ley. Se trata de un ente autárquico de derecho público y naturaleza multidisciplinaria que tiene a su cargo entre distintas cuestiones, la planificación, el registro, la fiscalización de las disposiciones del Código de Aguas y cualquier normativa que lo complemente o sustituya. Dicho código también define la figura del comité de cuenca, el cual funciona bajo la órbita de la ADA, debe estar integrado por un representante de cada municipio incluido en el área geográfica de su competencia, y le corresponde fijar las pautas para la preparación y ejecución de un programa de desarrollo integrado de la cuenca o región y atender su marcha. Así, la ADA concibe al comité de cuenca como la unidad de gestión territorial, cuyo objetivo es maximizar en forma equilibrada los beneficios ambientales, sociales y económicos de la cuenca.

En este sentido, y retomando el área de estudio, en la RMBA se encuentran conformados nueve comités de cuencas hidrográficas, como puede verse en el Cuadro 1. De todos ellos, quizás los que mayor importancia revistan sean los de la cuenca del Río Luján, del Reconquista y del Matanza-Riachuelo, dado que por la extensión territorial que abarcan involucran la participación de una gran cantidad de partidos, lo que indudablemente presenta un desafío aún mayor en lo que refiere a la gestión de cuencas. A su vez, cada entidad define sus propios objetivos y programas que, si bien tienen alguna influencia por parte de normativas e instrumentos de las escalas superiores, también es cierto que presentan sus particularidades.

\section{CUADRO 1}

Organismos de cuenca de la Región Metropolitana de Buenos Aires

\begin{tabular}{|l|l|}
\hline \multicolumn{1}{|c|}{ Organismos de cuenca } & \multicolumn{1}{c|}{ Municipios Integrantes } \\
\hline Comité de la cuenca hidrica del rio Luján & Moreno, José C. Paz, Malvinas Argentinas, Gral. Rodriguez, Tigre, Pilar, Escobar. \\
\hline Comité de la cuenca hidrica del rio Reconquista & $\begin{array}{l}\text { Tigre, San Fernando, San Isidro, Gral. San Martín, San Miguel, Malvinas } \\
\text { Argentinas, José C. Paz, Tres de Febrero, Hurlinghan, Ituzaingó, Morón, } \\
\text { Moreno, Merlo, Vicente López, General Rodriguez, Luján, Marcos Paz, Gral. Las }\end{array}$ \\
\hline Comité de la cuenca hídrica del Arroyo Medrano & San Martín, Tres de Febrero, Vicente López \\
\hline Autoridad de cuenca Matanza Riachuelo & $\begin{array}{l}\text { CABA, Lanús, Avellaneda, Lomas de Zamora, Esteban Echeverría, La Matanza, } \\
\text { Ezeiza, Cañuelas, Ate. Brown, Morón, Merlo, Marcos Paz, Presidente Perón, San } \\
\text { Vicente, Gral. Las Heras, PBA y Nación. }\end{array}$ \\
\hline Comité de la cuenca hidríca del Arroyo Galindez & Avellaneda, Lomas de Zamora, Lanús, Ate. Brown \\
\hline Comité de la cuenca hidrica del Arroyo Las Perdices & Avellaneda, Lomas de Zamora, Lanús, Ate. Brown \\
\hline Comité de cuenca hidrica del Arroyo San Francisco Las Piedras & Avellaneda, Lomas de Zamora, Florencio Varela, Quilmes \\
\hline Comité de la cuenca hidrica Vertiente Rio de La Plata Superior & Berazategui, Quilmes, Florencio Varela \\
\hline Comité de cuenca Región Capital & La Plata, Berisso, Ensenada \\
\hline
\end{tabular}

Fuente: Elaboración propia a partir de información de la Autoridad del Agua - PBA

El Comité de la Cuenca del Río Reconquista (COMIREC - Ley provincial 12.653/01) es un ente autárquico que tiene por objeto prestar servicios y realizar acciones conducentes a la gestión integral y preservación del recurso hídrico de la cuenca del río Reconquista, y que cuenta, a su vez, con la capacidad jurídica para planificar, coordinar, ejecutar y controlar la administración integral de la misma. En materia de 
ordenamiento territorial se destaca el Programa de Saneamiento Ambiental, el cual, entre diversas cuestiones, contempla acciones y obras para aumentar la cobertura de agua, alcantarillado y tratamiento de aguas residuales, mejorar la gestión de los residuos sólidos, el control y reconversión de industrias, el acceso a los barrios.

Por su parte, el Comité de Cuenca del Río Luján (COMILU - Ley provincial Nº14.710/15) tiene por objeto la realización de acciones tendientes a preservar el recurso hídrico y a gestionar el mismo de manera integral y sustentable. Entre otras cuestiones, está facultado para planificar, coordinar, ejecutar y controlar un plan de gestión integral y la administración integral de la cuenca. Esto lo habilita para planificar el ordenamiento territorial ambiental del territorio afectado a la cuenca, demarcar las líneas de ribera, definir la red de áreas protegidas a nivel de cuenca, entre otras facultades. Entre sus prioridades se encuentra la realización de obras tendientes a afectar la capacidad de conducción del río y el consecuente escurrimiento de las aguas, para así disminuir las zonas anegadas y la creación de un sistema de alerta temprana.

La Autoridad de Cuenca Matanza Riachuelo (ACUMAR) se distingue de los entes mencionados en los párrafos anteriores, en tanto se trata de una institución que nuclea no solo a distintos partidos de la provincia de Buenos Aires, sino también a representantes de CABA y del gobierno nacional. Creado en el año 2006 por disposición de la Ley nacional 26.168, como consecuencia del fallo de la Corte Suprema de Justicia de la Nación (CSJN) por la "causa Mendoza" (Merlinsky, 2016), el organismo tiene facultades de regulación, control y fomento respecto de las actividades industriales, la prestación de servicios públicos, y cualquier otra actividad con incidencia ambiental en la cuenca; puede intervenir administrativamente en materia de prevención, saneamiento, recomposición y utilización racional de los recursos naturales, y también en la planificación del ordenamiento ambiental del territorio afectado a la cuenca. Tiene como principio la participación social activa en tanto metodología de trabajo, la cual se basa en involucrar activamente a las organizaciones sociales en las mesas de trabajo, concebidas como instancias en las que múltiples actores abordan colectivamente problemáticas territoriales específicas. Impulsa, también por indicación de la CSJN, un plan integral de saneamiento ambiental, cuyos objetivos apuntan a mejorar la calidad de vida de los habitantes de la cuenca, recuperar el ambiente en todos sus componentes (agua, aire y tierra) y prevenir daños con suficiente y razonable grado de predicción, entre otras cuestiones.

\subsection{Ciudad Autónoma de Buenos Aires (CABA)}

El ordenamiento territorial en la Ciudad Autónoma de Buenos Aires se encuentra guiado por el Código Urbanístico aprobado en el año 2018 (su antecedente data de 1977). El mismo regula cuestiones relacionadas con el uso del suelo, la edificabilidad, el hábitat, la integración sociourbana, la protección patrimonial, entre otros aspectos que tienen relación con el ordenamiento urbanístico del territorio de la ciudad (Art. 1.1.2). En los principios de planificación urbana y ambiental que dan fundamento al Código (Art. 1.2.1) se mencionan cuestiones tales como: "la vinculación entre la Ciudad con los ríos que la rodean", "condiciones de habitabilidad que garanticen la calidad ambiental y la sostenibilidad", "provisión adecuada de infraestructura de saneamiento, prevención de inundaciones”. Más allá de eso, y a pesar de la presencia de numerosas cuencas, estas no son contempladas como unidades de gestión en el Código Urbanístico, en el que las únicas referencias vinculadas a la cuestión hídrica refieren a la prevención del riesgo hídrico (Artículos 7.2.8.1; 7.2.8.2; 7.2.8.3; y 7.2.8.3.1).

En este sentido, el continuo crecimiento y densificación de CABA tendió a entubar los distintos cursos de agua, para lo cual fue necesario el desarrollo de un complejo sistema de desagües que descargan principalmente en el Río de la Plata, y cuya construcción inició en el año 1873. Según consta en el sitio web del Gobierno de la Ciudad, estas obras fueron calculadas para una población y densidad edilicia mucho menores a las actuales, con una edificación abierta, espacios no impermeabilizados y bajo coeficiente de escorrentía y un cierto nivel de infiltración. Actualmente, casi la totalidad del agua que ingresa al sistema escurre sin tener espacios de 
retención o de infiltración. Sin embargo, dada la saturación del sistema, la escasa pendiente del terreno, la extensión de las cuencas y la intensidad y frecuencias de las tormentas, el drenaje rápido del agua presenta dificultades para la Ciudad, por lo que las inundaciones en distintos barrios son problemas frecuentes.

Por este motivo, CABA elaboró un plan director de ordenamiento hidráulico, pensado como un instrumento de diagnóstico (entre los años 2001 y 2006) de los problemas vinculados a las inundaciones para el posterior diseño de proyectos a ejecutar. En paralelo a este plan (y también como resultado del mismo), en el año 2005 el gobierno de CABA desarrolló un programa de gestión del riesgo hídrico (finalizado en 2013), el cual contemplaba obras en algunas de las cuencas de la Ciudad y el fortalecimiento en la prevención de inundaciones a partir de modificaciones en normativas, y campañas de educación y comunicación. La continuidad de estas acciones fue el objetivo de la creación del Plan Hidráulico en el año 2014, y el Proyecto de Asistencia a la Gestión de Riesgo de Inundaciones. Ante esto, algunos trabajos (González, 2018; Leveratto, 2019) plantean que, si bien hay un reconocimiento del problema, en la práctica persiste la tendencia a intentar resolverlo con obras hidráulicas que carecen de una mirada integral, ya que, en paralelo, se favorece una planificación urbana al servicio de unos pocos intereses, en tanto se permite la construcción en áreas de alta peligrosidad de inundación.

\section{REFLEXIONES FinALES}

Hasta aquí se intentó realizar una aproximación a los muy diversos instrumentos de gestión que tienen influencia en el ordenamiento territorial de la RMBA y específicamente en sus cuencas hidrográficas. Por tratarse de una indagación, es posible realizar algunos comentarios a modo de reflexiones finales, seguidos de preguntas disparadoras que permitirán profundizar el estudio en una instancia posterior.

En primer lugar, la revisión de los instrumentos permitió observar que aquellos referidos al manejo de cuencas vinculan dicho concepto a la conservación, manipulación, regulación, organización de los recursos hídricos, con un fuerte énfasis en la gestión del riesgo hídrico, la sostenibilidad y el saneamiento ambiental, cuestiones que van en línea con las concepciones más tradicionales. Por su parte, referido al ordenamiento territorial, en los instrumentos indagados se registra una repetición de términos tales como coordinación, equilibrio, inclusión, integración, armonización, que van en línea con los conceptos propuestos por las "miradas tradicionales" de ordenamiento territorial, vinculadas con las nociones de desarrollo, la política, la participación, modelo deseable y armonía.

Por otra parte, la complejidad ambiental y territorial de la RMBA, reconocida en numerosas investigaciones, pareciera no terminar de ser correctamente abordada por los instrumentos de gestión propuestos, dadas las múltiples jurisdicciones y el elevado grado de dispersión normativa existente. Las cuencas hidrográficas que allí se encuentran son reconocidas de manera diferencial por parte de estas jurisdicciones, por lo que su manejo adopta diversas formas (o ninguna, como en el caso de CABA). Se destaca, no obstante, la gran cantidad de comités de cuencas conformados, lo que evidencia que existen esfuerzos por crear espacios de gestión y consenso. Por lo expuesto, puede concluirse que, a grandes rasgos, el manejo de cuencas hidrográficas es efectivamente un mecanismo contemplado por el ordenamiento territorial en la RMBA, aunque su conceptualización e implementación difiere dependiendo de las escalas y jurisdicciones de los instrumentos de aplicación.

Para finalizar, cabría preguntarse cómo son abordados los conflictos ambientales en esta región, específicamente aquellos vinculados a las cuencas hidrográficas, y si efectivamente se cumplen los espacios de participación y debate que plantean los instrumentos y organismos con injerencia en este recorte territorial. Estas visiones podrían considerarse a priori como modelos ideales que, si bien reconocen la existencia de múltiples actores, la necesidad de favorecer al cuidado ambiental y a la integración social, tienden a desconocer o naturalizar los diferenciales de poder que cada actor ostenta en los procesos que dan origen a esos conflictos que buscan abordar. 
Algunas posibles líneas para continuar este estudio refieren a la indagación de más instrumentos de gestión, incluso con un mayor grado de profundización. El vasto universo normativo e institucional referido a las temáticas trabajadas con injerencia en el área de estudio supera ampliamente las páginas disponibles en un único artículo, por lo que quedará como tarea pendiente a desarrollar. Por otro lado, en estudios más particularizados será importante identificar los actores que participan y analizar su participación real, sus estrategias y capacidad de influir en las decisiones. Finalmente, resulta de interés también analizar la normativa e instrumentos de gestión a la luz de los modelos económicos y políticos en que se originan/ originaron, dado que los intereses cambian constantemente y el peso de unos y otros actores difiere de acuerdo al contexto y a la temática.

\section{Páginas Web consultadas:}

Consejo Federal de Planificación (COFEPLAN). Recuperado de https://www.argentina.gob.ar/habitat/c ofeplan

Consejo Hídrico Federal (COHIFE). Recuperado de https://www.cohife.org/

Gobierno de la Ciudad Autónoma de Buenos Aires (CABA). Recuperado de https://www.buenosaires.go b.ar/

Comité de Cuenca del Río Luján (COMILU). Recuperado de https://www.gba.gob.ar/comilu

Comité del Río Reconquista (COMIREC). Recuperado de https://www.gba.gob.ar/comirec

Autoridad de Cuenca Matanza Riachuelo (ACUMAR). Recuperado de https://www.acumar.gob.ar/

Autoridad del Agua (ADA). Recuperado de http://www.ada.gba.gov.ar/

Organismo Provincial para el Desarrollo Sostenible (OPDS). Recuperado de https://www.opds.gba.gov.ar/ Secretaría de Infraestructura y Política Hídrica. Recuperado de https://www.argentina.gob.ar/obras-public as/infraestructura-y-politica-hidrica

\section{REFERENCIAS}

Alvino, S. (2012). La gestión y el manejo de las cuencas hídricas en la Región Metropolitana de Buenos Aires. En M. Di Pace y A. Barsky (dir.), Agua y Territorio. Fragmentación y complejidad de la gestión del recurso hídrico en la Región Metropolitana de Buenos Aires (1 ed., pp. 101-121). Buenos Aires: Ciccus.

Arzeno, M. (2019). Orden-desorden y ordenamiento territorial como tecnología de gobierno. Revista de Geografía, 25(25), $1-16$.

Braz, A. M, Mirandola García, P. H., Pinto, A. L., Salinas Chávez, E., y de Oliveira, I. J. (2020). Manejo integrado de cuencas hidrográficas: posibilidades y avances en los análisis de uso y cobertura de la tierra. Cuadernos de Geografia, 29(1), 69-85. https://doi.org/10.15446/rcdg.v29n1.76232.

Cabrales Barajas, L. (2006). Geografía y Ordenamiento Territorial. En D. Hiernaux y A. Lindón (dir.), Tratado de Geografia Humana (pp. 601-627). México: Anthropos.

Calderón, G., Zulaica, M. L., Massone, H. E., y Dalla Torre, J. (2020). Vinculación entre el Ordenamiento Territorial y la gestión del agua en Argentina y en la provincia de Buenos Aires. Análisis de aspectos normativos e institucionales (2003-2019). Geografía Norte Grande, 77, 173-190. Recuperado de https://scielo.conicyt.cl/pd f/rgeong/n77/0718-3402-rgeong-77-173.pdf

Capuccio, S., y Mignaqui, I. (2014). Paradigmas Urbanísticos y Gestión Ambiental Metropolitana. Aportes de la Planificación Ecológica y sus posibilidades de aplicación en la Región Metropolitana de Buenos Aires. En $11^{\circ}$ Simposio de la Asociación Internacional de Planificación Urbana y Ambiente (UPE 11). La Plata: Universidad Nacional de La Plata. 
Carvajal Sánchez, N. I. (2011). Enfoques ascendente y descendente del ordenamiento territorial en Colombia. Cuadernos de Geografía, 20(1), 21-28. Recuperado de https://revistas.unal.edu.co/index.php/rcg/article/view/ $23064 / 36706$

Casaza, J. (2004). La situación del manejo de cuencas en la República Argentina. Informe Nacional. En III Congreso Latinoamericano de Manejo de Cuencas Hidrográficas. Arequipa, Perú.

Dourojeanni, A. y Jouravlev, A. (1999). Gestión de cuencas y ríos vinculados con centros urbanos. Comisión Económica para América Latina y el Caribe (CEPAL).

DPOUyT (2007). Lineamientos estratégicos para la Región Metropolitana de Buenos Aires. Alfredo Garay (Coord.). Secretaría de Urbanismo y Vivienda.

Farinos Dasí, J. (2014) Ordenación del territorio desde la geografía. De renovaciones conceptuales, retos, amenazas y espacios de oportunidad. Poligonos, 26, 7-58. Recuperado de http://dx.doi.org/10.18002/pol.v0i26.1698

Gómez Orea, D. (2003). La ordenación territorial: carácter, alcance y contenido. España: Universidad Politécnica de Madrid. Recuperado de https://core.ac.uk/reader/55526614

González, S., y Natenzon, C. (2002). Cuenca hidrográfica y riesgo ambiental. Dos estrategias de resolución de incertidumbre. Boletín de Estudios Geográficos, 97, 169-180.

González, S. G. (2018). Riesgo hídrico y planificación urbana en la ciudad de Buenos Aires. Estudios del hábitat, 16 (2) e047, pp. 1-13. Recuperado de https://revistas.unlp.edu.ar/Habitat/article/download/6026/5336/

Haesbaert, R. (2006). Ordenamento Territorial. Boletim Goiano de Geografia, 26(1), 117-124.

Haesbaert, R. (2014). Lógica zonal y ordenamiento territorial: Para rediscutir la proximidad y la contigüidad espaciales. Cultura y Representaciones Sociales, 16, 9-29. Recuperado de http://www.scielo.org.mx/scielo.php?script=sci_a rttext\&pid=S2007-81102014000100001

Hernández Peña, Y. T. (2010). El ordenamiento territorial y su construcción social en Colombia: ¿̇un instrumento para el desarrollo sustentable?. Cuadernos de Geografía, 19, 97-109.

Lanfanchi, G., Garay, A., Baer, L., y Bidart, M. (2017). Revisión de los Lineamientos Estratégicos para la Región Metropolitana de Buenos Aires 2007-2017. Documento de Trabajo N¹68. Buenos Aires: CIPPEC.

Leveratto, M. J. (2019). Cambio climático y planificación: ¿es el Nuevo Código Urbanístico una oportunidadperdida para Buenos Aires? (1 ${ }^{\circ} \mathrm{ed}$.). CABA: AS6030 Arquitectura y Sustentabilidad. Recuperado de https://as6030ar.files.w ordpress.com/2019/12/nuevo-codigo-urbanistico-caba-y-cambio-climatico_as6030_leveratto.pdf

Massiris Cabeza, A. (2005). Fundamentos conceptuales y metodológicos del ordenamiento territorial. Colombia: Universidad Pedagógica y Tecnológica de Colombia, Tunja. Recuperado de http://www.massiris.com/2012/0 9/libro-fundamentos-conceptuales-y.html

Merlinsky, G. (2006). Vulnerabilidad social y riesgo ambiental: ¿un plano invisible para las políticas públicas?. Mundo urbano, 28. Recuperado de http://www.mundourbano.unq.edu.ar/index.php/ano-2006/26-numero-28/189-1 -vulnerabilidad-social-y-riesgo-ambiental-un-plano-invisible-para-las-politicas-publicas

Merlinsky, G. (2016). Efectos de las causas estructurales en el largo plazo: la causa Riachuelo. Direito \& Práxis, 7(14), 397-420.

Montes Lira, P. (2001). El ordenamiento territorial como opción de politicas urbanas y regionales en América Latina y el Caribe. División de Medio Ambiente y Asentamientos Humanos, CEPAL. Serie Medio Ambiente y Desarrollo, 45, 59p.

Nené-Preciado, A. J., González Sansón, G., Mendoza, M. E., y Silva Bátiz, F. de A. (2017). Cambio de cobertura y uso de suelo en cuencas tropicales costeras del Pacífico central mexicano. Investigaciones Geográficas, 94, pp. 1-18. doi: https://dx.doi.org/10.14350/rig.56770

Oszlak, O., y O’Donnell, G. (1995). Estado y políticas en América Latina: hacia una estrategia de investigación. Redes, 2(4), 99-128.

Pulido, N. (2011). El crecimiento urbano en las cuencas de la región Andes de Venezuela y su articulación con el recurso agua. Revista Geográfica Venezolana, 52(2), 101-122. 
Reese, E. (2006). La situación actual de la gestión urbana y la agenda de las ciudades en la Argentina. Medio Ambiente y Urbanización, 65(1), 3-21.

Reese, E. (2014). Construcción del territorio, mercado y política. La experiencia de la ley de Acceso Justo al Hábitat Popular en la provincia de Buenos Aires. En D. M. Gargantini (Comp.), Hábitat, acceso al suelo y financiamiento. Experiencias alternativas de producción sociohabitacional (1ºd., pp. 13-17). Córdoba: Asociación Vivienda Económica.

Ríos, L. (2017). Capacidades estatales y políticas de ordenamiento territorial. Municipios de la provincia de Buenos Aires en el contexto pos-neoliberal (2003-2015). (Tesis de doctorado). FAU, UNLP. Recuperado de: http://hdl.hand le.net/10915/63911

Rotger, D. V. (2018). Gestión de cuencas en la región Metropolitana de Buenos Aires. Historia y actualidad de un territorio en conflicto ambiental. El caso del Gran La Plata. Cuaderno Urbano, 24(24), 7-26.

Ruiz Rivera, N., Casado Izquierdo, J. M., y Sánchez Salazar, M. T. (2015). Los Atlas de Riesgo municipales en México como instrumentos de ordenamiento territorial. Investigaciones Geográficas, 88, 146-162. Recuperado de http:/ /www.scielo.org.mx/pdf/igeo/n88/0188-4611-igeo-88-00146.pdf

Ugalde, V. (2010). La coordinación institucional del ordenamiento territorial en México. Revista de Geografía Norte Grande, 47, 105-120.

Zapperi, P.A. (2018). Análisis de la incorporación del riesgo de inundación en la normativa de ordenamiento territorial de Argentina. Investigaciones Geográficas, 70, 71-90. doi: https://doi.org/10.14198/INGEO2018.70.04

Zoido Naranjo, F. (1998). Geografía y ordenación del territorio. Scripta Vetera, 16, 19-31. Recuperado de http://w ww.ub.edu/geocrit/sv-77.htm 